\title{
Human Motion Video Analysis in Clinical Practice (Review)
}

DOI: $10.17691 / \mathrm{stm} 2015.7 .4 .26$

Received January 28, 2015

V.V. Borzikov, Junior Researcher, Scientific and Clinical Department;

N.N. Rukina, MD, PhD, Senior Researcher, Department of Functional Diagnosis;

O.V. Vorobyova, Junior Researcher, Department of Functional Diagnosis;

A.N. Kuznetsov, Junior Researcher, Department of Functional Diagnosis;

A.N. Belova, MD, DSc, Professor, Head of the Department of Functional Diagnosis

Privolzhsky Federal Research Medical Center, Ministry of Health of the Russian Federation,

18 Verkhne-Volzhskaya naberezhnaya, Nizhny Novgorod, 603155, Russian Federation

The development of new rehabilitation approaches to neurological and traumatological patients requires understanding of normal and pathological movement patterns. Biomechanical analysis of video images is the most accurate method of investigation and quantitative assessment of human normal and pathological locomotion. The review of currently available methods and systems of optical human motion analysis used in clinical practice is presented here. Short historical background is provided. Locomotion kinematics analysis using passive marker based systems is reviewed with special attention to the gait analysis. Clinical application of optical motion capture and analysis systems in the diagnosis of locomotion impairment, in Parkinson's disease with movement control disorders, stroke sequelae, monitoring of motor function rehabilitation in patients with infantile cerebral paralysis, limb joint endo- and exoprosthetics and some other disorders is described.

Key words: biomechanics; video analysis; optical human motion; rehabilitation medicine.

\section{Historical background}

The development of rehabilitation treatment technologies for patients with disease sequelae and traumas of the nervous system and muscularskeleton apparatus requires knowledge of locomotion mechanisms in norm and pathology, detailed analysis of motion disorder structure, and their quantitative assessment [1-4]. The quantitative study of human locomotions goes back to the first half of the XIX century, when Weber brothers published their investigation devoted to the time and spatial parameters of human motions [5]. Somewhat later a photographic technique was used to characterize the motion patterns $[6,7]$. In the same period, Brown, anatomist, and Fisher, mathematician, using the laws of Newton's mechanics, suggested the methods of calculating the force moments and energy consumption in the moving limb joints [8]. The development of force platforms and the study of walking kinetics became a real advance of the early $X X$ century [9]. An invaluable contribution to the study of locomotion was made by the founder of the Russian biomechanics Bernstein, who used kinocyclographic method to register displacements of human body joints by a stationary photocamera [10].

However, a wide study of locomotion became possible only with the advent of computer technologies [11-17]. Methods of locomotion kinematics assessment are based on the motion capture systems [18-20]. These systems can be magnetic (calculate the position and orientation of movement sensors with the help of magnetic fields and have low noise resistance), optical (analyze video images of the object) and inertial (movement biomechanics analysis is performed by receiving signals from the sensors of various modalities: goniometric, inclinometric, accelerometric, force, stretch sensors, etc., attached to the examined object) [21-28].

The biochemical analysis of movement video images remains the most precise method of human locomotion investigations $[29,30]$. The first video registering systems for locomotion kinematics study were developed at the end of the 70's of the XX century [31, 33]. This method implied fixing to the examinee's body actively reflective markers, working in the infrared spectrum and connected to the cameras by wires. A photosensitive matrix of the photo cameras transformed the registered infrared marker images to a digital row and recorded the mark coordinates to the computer RAM with the following graphic building of movement trajectories on the display and calculation of their parameters [34]. Modern systems of motion video analysis enable obtaining 3D images of locomotion and combine video analysis data with the analysis of signals received from other measuring devices such as a force platform, myograph, stabilographic platform [35, 36].

\section{Technology and methodology of movement kinematic study using video analysis}

Video analysis (motion capture) of movements is based on computer analysis of object's motion video images, video capturing being performed in a noncontact

For contacts: Rukina Nataliya Nikolaevna, e-mail: rukinann@mail.ru 
way, without cable connection of the registering unit with the object under study [15, 30, 37-39].

There exist two types of video motion capture systems - marker-based (markers or sensors are attached to the object's body), and markerless systems, based on computer vision and image recognition technologies [40-42]. Markers can be active (transmit information about their state, provide simplicity and accuracy of information processing, but often have a large size and, therefore, can make the motion of the object itself difficult) and passive (markers-reflectors) $[42,43]$. Markerless technologies of video capture analyze positional relationship of non-uniform parts of object image on the consecutive frames and require more complex calculations [43, 44].

At present video capture technologies, using passive markers, are more commonly used in clinical practice. Retro reflective sensors, the signals from which are registered by video cameras, are fixed to the examined object body; the data are transmitted to the computer, where information is processed on the basis of the specific computer model: movements of a real object are animated and a report, allowing the analysis of visually presented angular and linear kinematic characteristics of the studied motions, is formed [30]. Obtaining objective and useful information depends on the number of video cameras, construction, quantity and location of markers, building of the models meeting the investigation tasks, and selection of the software package for analytical data processing [36, 45, 46].

Passive markers, applied in biomechanical video, usually have spherical shape, small dimensions (10$15 \mathrm{~mm}$ in diameter) and small mass, they are covered with a reflecting (i.e. directionally reflecting the light) material $[42,47]$. Directed marker illumination is performed by the light-emitting diodes, located around camera objectives (infrared spectrum allows conducting investigations in the visible light); the light is reflected from the markers and returns to the camera objective with the subsequent detection of marker spatial coordinates.

To capture the position of the examined body segment in space it is necessary that several cameras work simultaneously. They should be located at various angles in such a way, that the object is within the field of their vision all the time [47].

There are 2D (analyzing object movement on the plane) and 3D (more popular) motion capture systems [48]. To gain 3D images there must be no less than 8 cameras; if more cameras are used, the accuracy increases, but the system becomes more laborconsuming and expensive. All cameras in image capture systems are synchronized, and controlled by a local computer network. The frequency of videorecording usually amounts to $100 \mathrm{~Hz}$ (100 frames per second), i.e. markers recognition is renewed 100 times/s, exceeding several times the frequency spectrum of angular movements in ground locomotion [43].
The room area for video analysis must be 100-150 $\mathrm{m}^{2}$ for each camera to form binocular field of vision [47]. Camera position relationship and coordinate system of the investigated zone are determined by a calibrating procedure (a reference marked object with the known geometric characteristics and location in space are used for this purpose).

The number of markers, attached to the body segments, depends on the task of the investigation. Not less than 3 markers must be always within the range of vision of minimum 3 cameras (as a plane is determined in space by three points), in real conditions significantly more markers are usually used to register the changes of the body segment position in movement and overlapping zones of different segment displacements. As a rule, the novel video capture systems are provided with readymade clusters of markers in the form of plates with four markers placed on them for long limb segments, and caps with markers for a head, etc. [40, 47, 49].

Recognition of the shape and position of the body segments on the basis of the information, obtained from the sensors is the most complicated step of video analysis [50]. There exist various approaches: with a model or without it. The most commonly used model is such, in which body segments (arm, forearm, pelvis, thigh, shin, foot, etc.) are conventionally considered as rigid (not changing their form) linear sections, the connection between which is made according to the principle "ball-socket" $[32,51]$. In the modern video capture systems the feasibility of building any 2D or 3D multilink investigation models is realized, enabling the researcher to display the segments of the locomotor system he is interested in $[29,52,53]$.

Before investigation calibration of the examinee is performed to "tie" individual dimensions of the registered body segments to the computer model. For this purpose calibrating markers, which are necessary for determination of physical dimensions of the examined segments, are placed on him. Ascheme of marker position and a 3D model of a man, built by a Simi Motion program (Germany) is presented on the Figure.

Calibrating markers are placed on bone projections of the body to indicate the borders of limbs or body segments; exact localization of these markers is determined by computer model applied and accepted standards [54].

The software of the modern video analysis systems provides initial kinematic data in compliance with the standards of the International Society of Biomechanics. These standards were developed by the Committee on standardization and terminology of this Society in order to provide comparability of the initial data of all biomechanical studies on human kinematics, presented by various researchers. Moreover, standards imply the use of terminology understandable for clinicians, making it possible to apply the results of biomechanical investigations in clinical practice [55]. 


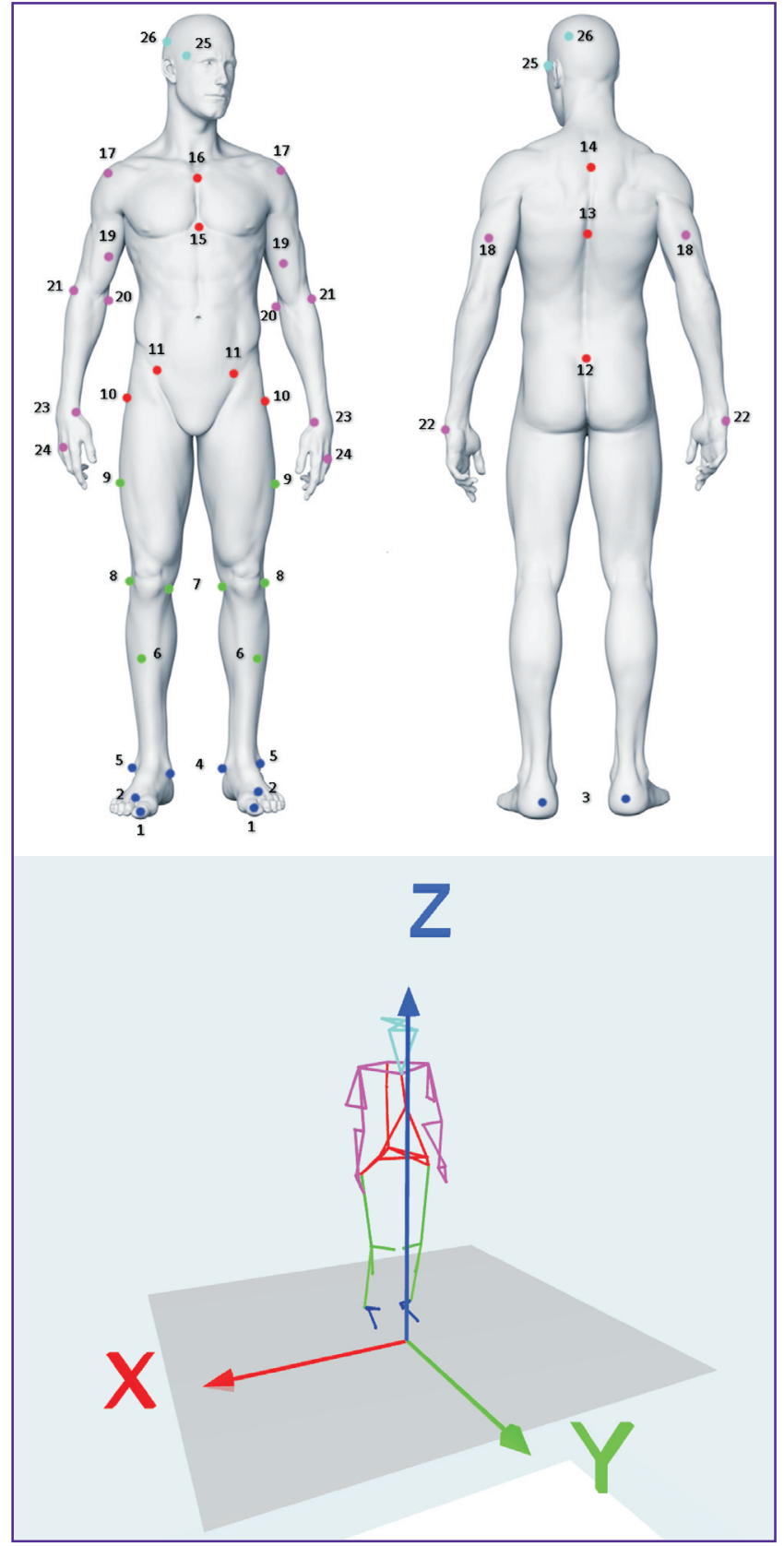

Marker position and 3D model of a man (Simi Motion, Germany)

Standards of biomechanical report on joint kinematics are based on the joint coordinate system, which was suggested as far as 1983 by Grood and Suntay for a knee joint [56]. Standards regulate terminology, bone reference points to define the body segment borders, types of movements and their coordinate system (axes). Presently such standards are developed for movement of the foot, ankle, thigh, pelvis, spine, hand, forearm, and arm $[57,58]$. For example, apex of the medial ankle, the most medially located point of the tibia lateral condyle, the most laterally located point of the tibia lateral condyle, tuberosity of tibia, the middle point between the medial and lateral ankles, the mid distance between the extreme points of the tibia medial and lateral condyles are anatomical reference points in the ankle joint used to describe the foot movement [58]. Taking into consideration the given bone reference points definitions of standard anatomical planes in the ankle joint (frontal, sagittal, transversal, torsion), neutral position in the ankle joint, coordinate system (exes) of movements in it are given [58].

During calibration tracking markers are placed on the examined body not only to determine the physical parameters of the examined body segment but to "bind" the location of the tracking markers to the given segment as well. When carrying on the study itself, the calibrating markers can be removed, since the information on the movement is based on the displacement of the tracking markers. The program processing of the tracking marker coordinates allows the analysis of the angular and linear kinematic characteristics of the investigated movements, building averaged profiles of the joint angles and angular velocities, calculation of standard deviations, performing comparative analysis of the results of several or one patient investigations in different periods of time, using reference variants for comparison $[59,60]$.

\section{Application of motion video analysis techniques in clinical practice}

Human motion video analysis is used in clinical practice to diagnose locomotive disorders and monitor the dynamics of motor function restoration in the course of treatment [61]. It enables the study of kinematics (spatial organization or the geometry of motions and their changes in time without consideration of the acting forces), while a combined application of video analysis, tensodynamometric platforms, and electromyography allows investigation of motion kinetics (various force and energy characteristics), the level of different muscle group activity, muscular interactions and synergy of the motional acts [47].

It is in the rehabilitation medicine that video analysis has the most significant influence on the quality of decision-making (optimization of kinesotherapy regimens, assessment of rehabilitation results for patients with infantile cerebral paralysis, or with stroke or craniocerebral trauma sequelae), in traumatology and orthopedics (making a decision on operative intervention, testing orthopedic equipment), in prosthetics and orthotics (selection of prostheses and supporting devices) [29, 30, 54].

Video analysis helps to study the patient's gait, movements of separate segments of upper or lower limbs depending on the tasks to be solved [46].

A "gold standard" in studying body movements and the most frequent type of locomotion, which is investigated with the help of video systems, is walking [47, 60-62]. A complete walking cycle (double-step period) for each leg is composed of limb support and 
limb transfer phases, and is limited by the period of time from the beginning of foot contact with the support to the start of the next contact of the same foot with the support [63-65]. During biomechanical videorecording a patient walks on the marked locomotor walkway along and outside of which video cameras are located. The object of the video must always be within the binocular field, i.e. must be seen simultaneously by the two adjacent cameras. The area of the walkway within the range of video camera coverage is called an active zone. The length of an active zone is determined by the length of double-steps: at least one double-step and about $50 \%$ of its length on both sides must be captured [66].

The character and pace of the examined walking (normal, with a support, with auxiliary devices, at a usual or other pace) are defined by the investigation tasks [67]. For example, analyzing walking at a free pace, an object is asked to go along the walkway with a usual speed and at a comfort pace; having reached the end of the walkway, he turns $180^{\circ}$ and goes to the other end of the walkway. In kinematic analysis of walking, movement of the body mass center and angular displacements in the low limb joints with computation of linear and angular velocities and accelerations, with determination of the shape and area of phase trajectories in the joints are defined [68].

Bioelectric activity of muscles involved in walking and a force reaction of the support are often recorded simultaneously, which makes it possible to study muscle interactions and synergy of the motional act.

Currently, various protocols and biomechanical models of video analysis of kinematics and kinetics of the body, pelvis, and lower limbs during walking are used, however, data obtained on their basis, as shown by comparative examination, correlate well with each other and, therefore, are suitable for comparison $[59,69]$.

Separate protocols are offered for children, enabling the minimization of examination time, obtaining also $3 \mathrm{D}$ data on the movements in the joints and segments of the lower limbs [70].

Revealing pathological patterns in walking is especially important on orthopedics and neurology. Video analysis is of great significance in evaluation of walking in patients with lower limb joint exoprostheses [71]. A detailed analysis of walking in such patients allows optimization of exoprosthesis designs [72]. However, video analysis of motions in people with limb prostheses has some difficulties, since anatomical parameters of these individuals do not comply with the average indices. This requires the development of special models of investigation, which would allow the adequate reflection of movements of patient's own limb and the prosthesis as well [48]. The strategy of creating such models for individuals with prostheses is complicated. The analysis of 51 investigations, devoted to this problem, showed, that the models used in them essentially differ from each other [48]. Thus, in more than $60 \%$ of investigations a traditional model was used, enabling the analysis of movement with three degrees of freedom, in $15 \%$ the model was able to analyze only linear motions, and in $19 \%$ it provided the analysis of movements with 6 degrees of freedom. The differences of the models used make it impossible comparison of data gained in various research centers. This demands the development of a unified strategy in the assessment of the results of prosthetic work by video analysis of motions.

Video motion capture technologies are used for gait assessment after prosthetic hip, ankle, and knee joint replacement, enabling the evaluation of lower limbs and pelvis kinematics in operated patients, stability of endoprosthesis when moving in different planes and with a load on lower limbs [71, 73]. Alradwan et al. [74] carried out a detailed analysis of kinematic data, obtained by video analysis in 58 patients with prosthetic hip replacement. The authors came to the conclusion, that video analysis may be an optimal method of evaluating endoprosthetics outcomes, though the development of a unified technology of such investigations and criteria of functional improvement assessment are necessary. Various characteristics of separate joints involved in walking may be studied with the help of video analysis, e.g. to examine instability in the ankle joint [75], movements in the knee joint [49].

In patients with stroke, gait video analysis can reveal pathological walking patterns and determine the directions of rehabilitation, supplementing traditional methods of biomechanical patient examination [67]. Kim et al. performed 3D analysis of kinematic curves at various walking speeds in 20 patients with hemiparesis, developed due to stroke. The investigator showed, that even in insignificant motion disorders, when a patient manages to maintain the walking speed comparable with the speed of healthy people, the pattern of walking changes greatly. Several typical patterns of pathological gait are described, the most intensive changes being registered in the frontal and sagittal planes, manifesting themselves primarily by the hyperfunction of the adductor muscles of the hip, insufficient bending in the knee and coxofemoral joints and reduction of the dorsal foot bending angle. The degree of disorders correlated with the walking speed. The authors prove convincingly, that a comprehensive analysis of 3D kinematic and kinetic gait profiles makes it possible to stimulate selectively the appropriate muscles and achieve a higher level of patient rehabilitation after stroke [76].

In case of nervous system damage with impaired movement control video analysis allows clarification of the character of motor activity disturbances typical for different neurological diseases [77, 78]. For example, having examined 113 patients with Parkinson's disease, Likhachev et al. [79] carried out a comparative analysis of purposeful movement indices, purposeless movement indices and coefficients of movement usefulness, gained 
during video capturing of functionally complicated step movement. The results of their research demonstrated global distortion of the motor program in Parkinson's disease and allowed to outline criteria of revealing objective conditions of basal ganglia damage in this disease. The same authors investigated video analytic profile of 34 patients with coordination impairments (multiple sclerosis, spinocerebellar degeneration) [80] and demonstrated that the assessment of coordination disorders by visual signs of video analytic profiles may be an adequate supplementation to the diagnosis of nervous system diseases. Video analysis is used for evaluation of movements of aged people [81], patients with Alzheimer's disease [78], diabetic people [82] in order to reveal gait changes occurring in these conditions.

In pediatric practice motion video analysis has found a wide application in rehabilitation of patients with infantile cerebral paralysis, enabling physicians to specify the character of walking disorders and to control the efficiency of the rehabilitating treatment. Titarenko et al. $[66,83,84]$ analyzed walking of children and adolescents with infantile cerebral paralysis in the form of spastic diplegia before and after "sensor therapy", including the application of reflex-loading device, multichannel programmed electro-stimulation of muscles in walking, functional biocontrol, etc. The authors revealed significant disorders of kinematics in these patients in the form of inversion of joint angle values and their correlations in various phases of the double-step cycle in comparison with the walk of healthy children. Video analysis of walking in infantile cerebral paralysis was shown to be able to assess the efficiency of rehabilitation and to perform biomechanical adjustment control of individual loading devices, used during rehabilitation therapy.

Motion video analysis makes it possible not only to evaluate walking, but also movements of the patients with some auxiliary appliances, which is critical for improving the design of those means, providing mobility of patients with limited walking abilities. For example, a methodology of video analysis of upper limb kinetics (location of the sensors, the model and protocol of investigation) in users of controlled invalid chairs has been developed [85]. Testing of this methodology on 23 patients with lower paraplegia showed, that different skills of controlling the chair are accompanied by various kinematics of upper limbs, and, respectively, by a different load on the muscles and joints of the upper limbs. The data obtained help to teach patients the correct skills of using the chair, and may also be useful in designing new chairs.

The feasibility of video analysis is not limited by gait investigation only, though methods of studying motions in the upper limb joints with the help of this technique are standardized to a lesser degree. Video analysis is likely to become invaluable means for objective assessment of hypermobility or, vice versa, limited movements in the shoulder joint [86, 87]. There appeared works, confirming the expediency of using 3D video analysis of movements in the shoulder joint as a method of controlling the efficiency of arthroplasty or prosthetic replacement of the shoulder joint $[88,89]$.

To develop methods of video analysis of hand movements is the most complicated task (due to a large number of joints and moving segments). Attempts have been made to synchronize acquisition of images using video analysis and computed tomography in order to improve the accuracy of recording angle movements [90].

\section{Prospects for future development}

Further development of motion video analysis technologies in clinical practice is connected with combined procedures of capture and analysis of kinematic and force movement characteristics, which requires synchronization of data gained from video analysis and other methods [91, 92]. Application of tensometric plates and inverse dynamic force transducers enables the assessment of support reaction force in walking and the values of torque for this or that movement in the joint, whereas the use of special programs makes it possible to calculate the expended mechanical energy [93-95]. These data are of special importance in rehabilitation medicine.

Markerless technologies of video analysis suitable for clinical practice are being further developed, helping to overcome such drawbacks of marker methods as disappearance of sensors from the field of camera vision when limbs turn, or disappearance of part of movement trajectory when markers are covered by the body segments [41, 42, 96].

Great attention is paid to the improvement of computer technologies of motion video analysis [97, 98], and creation of portable and more affordable systems [4, 99]. Video analysis of motions acquires a special role in connection with intensive developments being carried on in the field of robotic medical devices and systems [100].

Thus, motion video analysis enables clinicians to obtain important objective information, which helps to clarify the character of motor activity in various diseases of the musculoskeletal and nervous systems, and control the processes of rehabilitation. A rapid development of computer technologies will make the investigations of human locomotion more precise and less timeconsuming.

Study Funding. The work was supported by the Ministry of Education and Sciences of the Russian Federation within the framework of the Federal target program "Research and development in the priority directions of developing the scientific and technical complex of Russia for 2014-2020". Agreement on subsidy No.14.578.21.0094 of 24.11.2014 (unique project identifier RFMEFI57814X0094). 
Conflicts of Interest. The authors have no conflicts of interest.

\section{References}

1. Jonsson H., Kärrholm J. Three-dimensional knee joint movements during a step-up: evaluation after cruciate ligament rupture. J Orthoped Res 1994; 12(6): 769-779, http://dx.doi. org/10.1002/jor.1100120604.

2. Gavrila D.M. The visual analysis of human movement: a survey. Computer Vision and Image Understanding 1999; 73(1): 82-98, http://dx.doi.org/10.1006/cviu.1998.0716.

3. Likhachev S.A., Lukashevich V.A. To the question of methods of motion video analysis application. Meditsinskie novosti 2008; 12: 38-44.

4. Krishnan C., Washabaugh E.P., Seetharaman Y. A low cost real-time motion tracking approach using webcam technology. $J$ Biomech 2015; 48(3): 544-548, http://dx.doi.org/10.1016/ j.jbiomech.2014.11.048.

5. Weber W., Weber E. Mechanik der menschlichen Gehwerkzeuge. Göttingen: Dieterich; 1836.

6. Marey E. Animal mechanism: a treatise on terrestrial and aerial locomotion. London: Henry S. King \& Co.; 1874, http:// dx.doi.org/10.5962/bhl.title.84571.

7. Muybridge E. Animal locomotion. Philadelphia: J.B. Lippincott Company; 1887.

8. Braune W., Fischer O. Determination of the moments of inertia of the human body and its limbs. Springer-Verlag Berlin Heidelberg; 1988, http://dx.doi.org/10.1007/978-3-662-11236-6.

9. Baker R. The history of gait analysis before the advent of modern computers. Gait Posture 2007; 26(3): 331-342, http:// dx.doi.org/10.1016/j.gaitpost.2006.10.014.

10. Bernshteyn N.A. O postroenii dvizheniy [About construction of movements]. Moscow: Gosudarstvennoe izdatel'stvo meditsinskoy literatury; 1947; $254 \mathrm{p}$.

11. Romanov D.A. Upravlenie tekhnicheskoy podgotovlennost'yu sportsmenov na osnove komp'yuternogo videoanaliza dvizheniy. Dis. ... kand. ped. nauk [Management of sportsmen technical performance using computer-based motion video analysis. PhD Thesis]. Krasnodar; 2004.

12. Cedras C., Shah M. Motion-based recognition a survey. Image and Vision Computing 1995; 13(2): 129-155, http://dx.doi. org/10.1016/0262-8856(95)93154-k.

13. Gavrila D.M., Davis L.S. 3-D model-based tracking of humans in action: a multi-view approach. Proceedings CVPR IEEE Computer Society Conference on Computer Vision and Pattern Recognition 1996; p. 73-80, http://dx.doi.org/10.1109/ cvpr.1996.517056.

14. Furnée H. Real-time motion capture systems. In: Allard $P$., Cappozzo A., Lundberg A., Vaughan C.L. (editors). Threedimensional analysis of human locomotion. Chichester, UK: John Wiley \& Sons; 1997; p. 85-108.

15. Human motion analysis: current applications and future directions. Harris G.F., Smith P.A. (editors). New York: IEEE Press; 1996.

16. Wren C.R., Azarbayejani A., Darrell T., Pentland A.P. Pfinder: real-time tracking of the human body. IEEE Transactions on Pattern Analysis and Machine Intelligence 1997; 19(7): 780785, http://dx.doi.org/10.1109/34.598236.

17. Kakadiaris I.A., Metaxas D. 3D human body model acquisiton from multiple views. Proceedings of IEEE International Conference on Computer Vision 1995, http://dx.doi.org/10.1109/ iccv.1995.466881.
18. Narayanan P.J., Rander P., Kanade T. Technical Report CMU-RI-TR-95-25. Robotics Institute Carnegie Mellon University; 1995. Synchronous capture of image sequences from multiple cameras.

19. Karaulova I.A., Hall P.M., Marshall A.D. Tracking people in three dimensions using a hierarchical model of dynamics. Image and Vision Computing 2002; 20: 691-700, http://dx.doi. org/10.1016/s0262-8856(02)00059-8.

20. Sigal L., Balan A.O., Black M.J. HumanEva: synchronized video and motion capture dataset and baseline algorithm for evaluation of articulated human motion. International Journal of Computer Vision 2009; 87(1-2): 4-27, http://link.springer.com/ article/10.1007\%2Fs11263-009-0273-6.

21. Hogg D. Model-based vision: a program to see a walking person. Image and Vision Computing 1983; 1(1): 5-20, http:// dx.doi.org/10.1016/0262-8856(83)90003-3.

22. Lee H.J., Chen Z. Determination of $3 D$ human body posture from a single view. Computer Vision, Graphics, and Image Processing 1985; 29(3): 396, http://dx.doi.org/10.1016/0734189x(85)90137-9.

23. Lafortune M.A., Cavanagh P.R., Sommer H.J., Kalenak A. Three-dimensional kinematics of the human knee during walking. J Biomechanics 1992; 25(4): 347-357, http://dx.doi. org/10.1016/0021-9290(92)90254-x.

24. de Vries W.H.K., Veeger H.E.J., Baten C.T.M., van der Helm F.C.T. Magnetic distortion in motion labs, implications for validating inertial magnetic sensors. Gait Posture 2009; 29(4): 535-541, http://dx.doi.org/10.1016/j.gaitpost.2008.12.004.

25. Engin M., Demirel A., Engin E.Z., Fedakar M. Recent developments and trends in biomedical sensors. Measurement 2005; 37(2): 173-188, http://dx.doi.org/10.1016/j.measurement.2 004.11.002.

26. Ghoussayni S., Stevens C., Durham S., Ewins D. Assessment and validation of a simple automated method for the detection of gait events and intervals. Gait Posture 2004; 20(3): 266-272, http://dx.doi.org/10.1016/j.gaitpost.2003.10.001.

27. Kwon D.Y., Gross M. Combining body sensors and visual sensors for motion training. Proceedings of the 2005 ACM SIGCHI International Conference on Advances in Computer Entertainment Technology; Valencia, Spain 15-17 Jun 2005; p. 94-101, http:// dx.doi.org/10.1145/1178477.1178490.

28. Tao W., Liu T., Zheng R., Feng $H$. Gait analysis using wearable sensors. Sensors (Basel) 2012; 12(2): 2255-2283, http://dx.doi.org/10.3390/s120202255.

29. Andriacchi T.P., Alexander E.J. Studies of human locomotion: past, present and future. J Biomech 2000; 33(10): 1217-1224, http://dx.doi.org/10.1016/S0021-9290(00)00061-0.

30. Moeslund T.B., Granum E. A survey of computer visionbased human motion capture. Computer Vision and Image Understanding 2001; 81(3): 231-268, http://dx.doi.org/10.1006/ cviu.2000.0897.

31. Andriacchi T.P., Ogle J.A., Galante J.O. Walking speed as a basis for normal and abnormal gait measurements. J Biomech 1977; 10(4): 261-268, http://dx.doi.org/10.1016/00219290(77)90049-5.

32. Spoor C.W., Veldpaus F.E. Rigid body motion calculated from spatial co-ordinates of markers. J Biomech 1980; 13(4): 391393, http://dx.doi.org/10.1016/0021-9290(80)90020-2.

33. Ferrigno G., Pedotti A. Elite: a digital dedicated hardware system for movement analysis via real-time TV signal processing. IEEE Trans Biomed Eng 1985; 32(11): 943-950, http://dx.doi. org/10.1109/tbme.1985.325627.

34. Dotsenko V.I., Voronov A.V., Titarenko N.Yu., 
Titarenko K.E. Computer-based motion video analysis in sports medicine and neurorehabilitation. Meditsinskiy alfavit 2005 3(41): 12-14.

35. Cappozzo A., Della Croce U., Leardini A., Chiari L. Human movement analysis using stereophotogrammetry. Part 1: theoretical background. Gait Posture 2005; 21(2): 186-196, http:// dx.doi.org/10.1016/j.gaitpost.2004.01.010.

36. Wang L., Hu W., Tan T. Recent developments in human motion analysis. Pattern Recognition 2003; 36(3): 585-601, http:// dx.doi.org/10.1016/s0031-3203(02)00100-0.

37. Isard M., Blake A. Visual tracking by stochastic propagation of conditional density. In: Proceeding of the 4th European Conference on Computer Vision. New York: 1996; p. 343-356.

38. Bregler C., Malik J. Tracking people with twists and exponential maps. Proceedings 1998 IEEE Computer Society Conference on Computer Vision and Pattern Recognition 1998, http://dx.doi.org/10.1109/cvpr.1998.698581.

39. Ma Y., Soatto S., Košecká J., Sastry S. An invitation to 3D vision. Interdisciplinary Applied Mathematics. Springer New York; 2004, http://dx.doi.org/10.1007/978-0-387-21779-6.

40. Cappozzo A., Cappello A., Croce U.D., Pensalfini F. Surface-marker cluster design criteria for 3-D bone movement reconstruction. IEEE Trans Biomed Eng 1997; 44(12): 1165-1174, http://dx.doi.org/10.1109/10.649988.

41. Ceseracciu E., Sawacha Z., Cobelli C. Comparison of markerless and marker-based motion capture technologies through simultaneous data collection during gait: proof of concept. PLoS One 2014; 9(3): e87640, http://dx.doi.org/10.1371/journal. pone.0087640.

42. Mündermann L., Corazza S., Andriacchi T. The evolution of methods for the capture of human movement leading to markerless motion capture for biomechanical applications. J Neuroeng Rehabil 2006; 3: 6, http://dx.doi.org/10.1186/1743-0003-3-6.

43. Vlasenko V.P. Tekhnologiya "Motion Capture". Periferiynye ustroystva [Technology "Motion Capture". Peripherals]. Zaporizhia; 2007. URL: http://www.bestreferat.ru/referat-401678.html.

44. Lanshammar H., Persson T., Medved V. Comparison between a marker-based and a marker-free method to estimate centre of rotation using video image analysis. In: Second World Congress of Biomechanics. Amsterdam; 1994.

45. Besl P., McKay N. A method for registration of 3-D shapes. IEEE Transactions on Pattern Analysis and Machine Intelligence 1992; 14(2): 239-256, http://dx.doi.org/10.1109/34.121791.

46. Aggarwal J., Cai Q. Human motion analysis: a review. Computer Vision and Image Understanding 1999; 73(3): 428-440, http://dx.doi.org/10.1006/cviu.1998.0744.

47. Skvortsov D.V. The methods of investigation of kinematics and modern standards. Videoanalysis. Lechebnaya fizkul'tura $i$ sportivnaya meditsina 2012; 12: 4-10.

48. Kent J., Franklyn-Miller A. Biomechanical models in the study of lower limb amputee kinematics: a review. Prosthet Orthot Int 2011; 35(2): 124-39, http://dx.doi.org/10.1177/030936461140 7677.

49. Andriacchi T.P., Alexander E.J., Toney M.K., Dyrby C., Sum J. A point cluster method for in vivo motion analysis: applied to a study of knee kinematics. J Biomech Eng 1998; 120(6): 743749, http://dx.doi.org/10.1115/1.2834888.

50. Zakotnik J., Matheson T., Dürr V. A posture optimization algorithm for model-based motion capture of movement sequences. J Neurosci Methods 2004; 135(1-2): 43-54, http:// dx.doi.org/10.1016/j.jneumeth.2003.11.013.

51. Lu T.-W., O'Connor J.J. Bone position estimation from skin marker coordinates using global optimization with joint constraints.
J Biomech 1999; 32(2): 129-134, http://dx.doi.org/10.1016/s00219290(98)00158-4.

52. Herda L., Fua P., Plänkers R., Boulic R., Thalmann D. Using skeleton-based tracking to increase the reliability of optical motion capture. Hum Mov Sci 2001; 20(3): 313-341, http://dx.doi. org/10.1016/s0167-9457(01)00050-1.

53. Royo Sánchez A.C., Aguilar Martín J.J., Santolaria Mazo J. Development of a new calibration procedure and its experimental validation applied to a human motion capture system. J Biomech Eng 2014; 136(12): 124502, http://dx.doi. org/10.1115/1.4028523.

54. Romkes J., Rudmann C., Brunner R. Changes in gait and EMG when walking with the Masai Barefoot Technique. Clin Biomech 2006; 21(1): 75-81, http://dx.doi.org/10.1016/j.clinbiome ch.2005.08.003.

55. Wu G., Cavanagh P. ISB recommendation for standardization in the reporting of kinematic data. $J$ Biomech 1995; 28(10): 1257-1261, http://dx.doi.org/10.1016/00219290(95)00017-c.

56. Grood E.S., Suntay W.J. A joint coordinate system for the clinical description of three-dimensional motions: application to the knee. J Biomech Eng 1983; 105(2): 136-144, http://dx.doi. org/10.1115/1.3138397.

57. Wu G., van der Helm F.C., Veeger H.E., Makhsous M., Van Roy P., Anglin C., Nagels J., Karduna A.R., McQuade K., Wang X., Werner F.W., Buchholz B. ISB recommendation on definitions of joint coordinate systems of various joints for the reporting of human joint motion - part II: shoulder, elbow, wrist and hand. J Biomech 2005; 38(5): 981-992, http://dx.doi.org/10.1016/ j.jbiomech.2004.05.042.

58. Wu G., Siegler S., Allard P., Kirtley C., Leardini A., Rosenbaum D., Whittle M., D'Lima D.D., Cristofolini L., Witte H., Schmid O., Stokes I. ISB recommendation on definitions of joint coordinate system of various joints for the reporting of human joint motion - part I: ankle, hip, and spine. J Biomech 2002; 35(4): 543-548.

59. Gorton G.E., Hebert D.A., Gannotti M.E. Assessment of the kinematic variability among 12 motion analysis laboratories. Gait and Posture 29(3): 398-402, http://dx.doi.org/10.1016/ j.gaitpost.2008.10.060.

60. Eng J.J., Winter D.A. Kinetic analysis of the lower limb during walking: what information can be gained from a threedimensional model? J Biomech 1995; 28(6): 753-758, http:// dx.doi.org/10.1016/0021-9290(94)00124-m.

61. Simon R.S. Quantification of human motion: gait analysisbenefits and limitations to its application to clinical problems. J Biomech 2004; 37(12): 1869-1880, http://dx.doi.org/10.1016/ j.jbiomech.2004.02.047.

62. Chau T. A review of analytical techniques for gait data. Part 1: fuzzy, statistical and fractal methods. Gait Posture 2001; 13(1): 49-66, http://dx.doi.org/10.1016/S0966-6362(00)00094-1.

63. Inman V., Ralston H., Todd F. Human walking. Baltimore: Williams \& Wilkins; 1981.

64. Perry J., Thorofare S., Jon D. Gait analysis: normal and pathological function. JAMA 1992; 268(22): 3257, http://dx.doi. org/10.1097/01241398-199211000-00023.

65. Winter D.A. Kinematic and kinetic patterns in human gait: variability and compensating effects. Hum Mov Sci 1984; 3(1-2): 51-76, http://dx.doi.org/10.1016/0167-9457(84)90005-8.

66. Voronov A.V., Dotsenko V.I., Titarenko K.E., Titarenko N.Yu. Komp'yuternyy videoanaliz dvizheniy v nauchnykh issledovaniyakh i klinicheskoy praktike. V kn.: Sotsial'naya pediatriya: sbornik nauchnykh trudov [Computer-based motion video analysis in 
scientific researches and clinical practice. In: Social pediatrics: collection of scientific proceedings]. Kiev; 2005.

67. Stokic D.S., Horn T.S., Ramshur J.M., Chow J.W. Agreement between temporospatial gait parameters of an electronic walkway and a motion capture system in healthy and chronic stroke populations. Am J Phys Med Rehabil 2009; 88(6): 437-444, http://dx.doi.org/10.1097/PHM.0b013e3181a5b1ec.

68. Whittle M.W., Barnes S.C. Defining normal ranges for gait parameter. In: Gait Anal Med Photogramm. Vol. 1-3. Oxford, Headington; 1987; p. 46-47.

69. Ferrari A., Benedetti M.G., Pavan E., Frigo C., Bettinelli D., Rabuffetti M., Crenna P., Leardini A. Quantitative comparison of five current protocols in gait analysis. Gait Posture 2008; 28(2): 207-216, http://dx.doi.org/10.1016/j.gaitpost.2007.11.009.

70. Leardini A., Sawacha Z., Paolini G., Ingrosso S., Nativo R., Benedetti M.G. A new anatomically based protocol for gait analysis in children. Gait Posture 2007; 26(4): 560-571, http://dx.doi. org/10.1016/j.gaitpost.2006.12.018.

71. Zhao S., Chen Y.S., Zhang X.L. Clinical application of gait analysis in hip arthroplasty. Orthop Surg 2010; 2(2): 94-99, http:// dx.doi.org/10.1111/j.1757-7861.2010.00070.x.

72. Lawson B.E., Huff A., Goldfarb M. A preliminary investigation of powered prostheses for improved walking biomechanics in bilateral transfemoral amputees. Conf Proc IEEE Eng Med Biol Soc 2012; 2012: 4164-4167, http://dx.doi. org/10.1109/EMBC.2012.6346884.

73. Mclnnes K.A., Younger A.S., Oxland T.R. Initial instability in total ankle replacement: a cadaveric biomechanical investigation of the STAR and agility prostheses. J Bone Joint Surg Am 2014; 96(17): e147, http://dx.doi.org/10.2106/JBJS.L.01690.

74. Alradwan H., Khan M., Grassby M.H., Bedi A., Philippon M.J., Ayeni O.R. Gait and lower extremity kinematic analysis as an outcome measure after femoroacetabular impingement surgery. Arthroscopy 2015; 31(2): 339-344, http:// dx.doi.org/10.1016/j.arthro.2014.06.016.

75. Gribble P., Robinson R. Alterations in knee kinematics and dynamic stability associated with chronic ankle instability. $J$ Athl Train 2009; 44(4): 350-355, http://dx.doi.org/10.4085/1062-605044.4.350.

76. Kim C.M., Eng J.J. Magnitude and pattern of 3D kinematic and kinetic gait profiles in persons with stroke: relationship to walking speed. Gait Posture 2004; 20(2): 140-146, http://dx.doi. org/10.1016/j.gaitpost.2003.07.002.

77. Das S., Trutoiu L., Murai A., Alcindor D., Oh M., De la Torre F., Hodgins J. Quantitative measurement of motor symptoms in Parkinson's disease: a study with full-body motion capture data. Conf Proc IEEE Eng Med Biol Soc 2011; 2011: 6789-6792, http://dx.doi.org/10.1109/IEMBS.2011.6091674.

78. Cedervall Y., Halvorsen K., Aberg A.C. A longitudinal study of gait function and characteristics of gait disturbance in individuals with Alzheimer's disease. Gait Posture 2014; 39(4): 1022-1027, http://dx.doi.org/10.1016/j.gaitpost.2013.12.026.

79. Likhachev S.A., Lukashevich V.A., Khromenkov A.V. The video motion analysis as a method of the diagnosis of the basal ganglion lesion in Parkinson's disease. Zhurnal nevrologii $i$ psikhiatrii im. S.S. Korsakova 2011; 111(12): 44-47.

80. Likhachov S.A., Lukashevich V.A. Videoanalysis of step movement: phenomenology of visual estimation. Mezhdunarodnyy nevrologicheskiy zhurnal 2012; 2: 178-182.

81. Jerome G.J., Ko S.U., Kauffman D., Studenski S.A., Ferrucci L., Simonsick E.M. Gait characteristics associated with walking speed decline in older adults: results from the Baltimore Longitudinal Study of Aging. Arch Gerontol Geriatr
2015; 60(2): 239-243, http://dx.doi.org/10.1016/j.archger.2015. 01.007.

82. Sawacha Z., Gabriella G., Cristoferi G., Guiotto A., Avogaro A., Cobelli C. Diabetic gait and posture abnormalities: a biomechanical investigation through three dimensional gait analysis. Clin Biomech (Bristol, Avon) 2009; 24(9): 722-728, http:// dx.doi.org/10.1016/j.clinbiomech.2009.07.007.

83. Titarenko N.Yu., Voronov A.V., Semenova K.A., Dotsenko V.I., Titarenko K.E., Levchenkova V.D., Politova I.Ya. Computer-based motion video analysis in the assessment of rehabilitation treatment of children with residual neuromotor deficiency. Funktsional'naya diagnostika 2006; 3: 69-75.

84. Titarenko N.lu., Voronov A.V. The effect of the reflex-load device Gravistat/Graviton on walk stereotype in patients with spastic diplegia. Zhurnal nevrologii i psikhiatrii im. S.S. Korsakova 2012; 111(7-2): 18-23.

85. Tsai C.Y., Hogaboom N.S., Boninger M.L., Koontz A.M. The relationship between independent transfer skills and upper limb kinetics in wheelchair users. Biomed Res Int 2014; 2014: 984526, http://dx.doi.org/10.1155/2014/984526.

86. Ropars M., Cretual A., Thomazeau H., Kaila R., Bonan I. Volumetric definition of shoulder range of motion and its correlation with clinical signs of shoulder hyperlaxity. A motion capture study. $J$ Shoulder Elbow Surg 2015; 24(2): 310-316, http://dx.doi. org/10.1016/j.jse.2014.06.040.

87. Crétual A., Bonan I., Ropars M. Development of a novel index of shoulder's mobility based on the configuration space volume and its link to mono-axial amplitudes. Man Ther 2015; 20(3): 433-439, http://dx.doi.org/10.1016/j.math.2014.10.020.

88. Maier M.W., Kasten P., Niklasch M., Dreher T., Zeifang F., Rettig O., Wolf S.I. 3D motion capture using the HUX model for monitoring functional changes with arthroplasty in patients with degenerative osteoarthritis. Gait Posture 2014; 39(1): 7-11, http:// dx.doi.org/10.1016/j.gaitpost.2013.07.111.

89. Hebert J.S., Lewicke J., Williams T.R., Vette A.H. Normative data for modified Box and Blocks test measuring upperlimb function via motion capture. J Rehabil Res Dev 2014; 51(6): 918-932, http://dx.doi.org/10.1682/JRRD.2013.10.0228.

90. Buffi J.H., Sancho Bru J.L., Crisco J.J., Murray W.M. Evaluation of hand motion capture protocol using static computed tomography images: application to an instrumented glove. J Biomech Eng 2014; 136(12): 124501, http://dx.doi. org/10.1115/1.4028521.

91. Jagos H., Oberzaucher J., Reichel M., Zagler W.L., Hlauschek W. A multimodal approach for insole motion measurement and analysis. Procedia Eng 2010; 2(2): 3103-3108, http://dx.doi.org/10.1016/j.proeng.2010.04.118.

92. Miller A.L. A new method for synchronization of motion capture and plantar pressure data. Gait Posture 2010; 32(2): 279381, http://dx.doi.org/10.1016/j.gaitpost.2010.04.012.

93. Martin C., Bideau B., Bideau N., Nicolas G., Delamarche P., Kulpa R. Energy flow analysis during the tennis serve: comparison between injured and noninjured tennis players. Am J Sports Med 2014; 42(11): 2751-2760, http://dx.doi.org/10.1177/036354651454 7173.

94. Raychoudhury S., Hu D., Ren L. Three-dimensional kinematics of the human metatarsophalangeal joint during level walking. Front Bioeng Biotechnol 2014; 2: 73, http://dx.doi. org/10.3389/fbioe.2014.00073.

95. Seel T., Raisch J., Schauer T. IMU-based joint angle measurement for gait analysis. Sensors (Basel) 2014; 14(4): 6891-6909, http://dx.doi.org/10.3390/s140406891.

96. Corazza S., Mündermann L., Chaudhari A., Demattio T., 
Cobelli C., Andriacchi T.P. A markerless motion capture system to study musculoskeletal biomechanics: visual hull and simulated annealing approach. Ann Biomed Eng 34(6): 1019-1029, http:// dx.doi.org/10.1007/s10439-006-9122-8.

97. Lenar J., Witkowski M., Carbone V., Kolk S., Adamázyk M., Sitnik R., van der Krogt M., Verdonschot N. Lower body kinematics evaluation based on a multidirectional four-dimensional structured light measurement. J Biomed Opt 2013; 18(5): 56014, http://dx.doi. org/10.1117/1.JBO.18.5.056014.

98. Belyea B.C., Lewis E., Gabor Z., Jackson J., King D.L. Validity and intra-rater reliability of 2-dimensional motion analysis using a hand-held tablet compared to traditional 3-dimensional motion analysis. J Sport Rehabil 2015, http://dx.doi.org/10.1123/ jsr.2014-0194.

99. Bonnet V., Sylla N., Cherubini A., Gonzáles A., Azevedo Coste C., Fraisse P., Venture G. Toward an affordable and userfriendly visual motion capture system. Conf Proc IEEE Eng Med Biol Soc 2014; 2014: 3634-3637, http://dx.doi.org/10.1109/ EMBC.2014.6944410.

100. Lin H.-I., Lin Y.-H. A novel teaching system for industrial robots. Sensors (Basel) 2014; 14(4): 6012-6031, http://dx.doi. org/10.3390/s140406012. 